TRANSPORTE LITORAL SOBRE LAS BARRAS DE GUERRERO NEGRO Y OJO DE LIEBRE.

por

Silvio Guido L. Marinone

Centro de Investigación Científica y de Educación Superior de Ensenada, B.C.

Espinoza No. 843

Ensenada, Baja California, México.

y

Román Lizārraga Arciniega

Instituto de Investigaciones Oceanológicas

Apartado Postal 453

Ensenada, Baja California, México.

RESUMEN

Se.presenta una evaluación del transporte potencial de arena sobre las barras de las lagunas de Guerrero Negro y Ojo de Liebre. El cálculo se hace en función del $69 \%$ de ocurrencia del oleaje de más energía proveniente del hemisferio norte. Se encontró que el transporte litoral neto paralelo a la playa es del orden de $6 \times 10^{4} \mathrm{~m}^{3} / \mathrm{anno}$ en direcciôn noreste. El sentido de este transporte es el mismo que el de la migración de la boca de la laguna de Guerrero Negro reportada por Phleger (1965).

\title{
ABSTRACT
}

An evaluation of the potential littoral sand transport on the beaches of Guerrero Negro and Ojp de Liebre Lagoons is presented. Calculations were made taking into account $69 \%$ of the highest energy waves from the Northern Hemisphere. It was found that the net transport of sand along the beach is on the order of $6 \times 10^{4} \mathrm{~m}^{3} /$ year to the NE. This transport is in the same direction as the migration of the mouth of Guerrero Negro Lagoon (Phleger, 1965). 


\section{INTRODUCC ION}

E1 sistema litoral es una zona en la que existen continuos cambios, muchos de los cuales son producidos por la actividad humana en beneficio o perjuicio de la zona 1itoral.

La playa como elemento del sistema, responde activamente a los factores físicos como oleaje y viento, reflejândose en el avance o retroceso de la linea de playa. Esta variaciơn es, por lo tanto, el resultado del contínuo cambio de volúmen de arena en una secciôn determinada. La cuantificación y/o predicción del movimiento de arena ocupa un lugar importante en la planeación y utlización de la zona costera.

El transporte de arena en el sistema litoral está definido por dos componentes: A) acarreo paralelo a la playa y B) acarreo perpendicular a la misma. Ambos pueden ser bidireccionales en un mismo punto en diferentes tiempos.

En el extenso 1itoral de Baja California se cuenta con determinaciones de transporte litoral a lo largo de la playa muy limitadas espacialmente, tanto en el Golfo de California (Komar e Inman, 1970; Komar, 1971) como en el Pacífico (O'brien y Zeevart, 1968; Lizárraga-Arciniega, 1972).

E1 objetivo de este trabajo es estimar 1a razón de transporte potencial de arena (paralelo a la playa) en el área de las barras ojo de Liebre y San José en la Bahía Vizcaíno, Baja California. Este cálculo se hace en base a parámetros de oleaje (altura de la ola, $\mathrm{H}$; perído, $\mathrm{T}$; ángulo de aproximación, a) cuyo origen y fuente de generación es el hemisferio norte.

AREA DE ESTUDIO

Las lagunas de Ojo de Liebre y Guerrero Negro forman parte del complejo lagunero en la costa del Pacifico situado entre los $27^{\circ} 35^{\prime}$ y $28^{\circ} 15^{\prime} \mathrm{N}$ y los $113^{\circ} 50^{\prime}$ y $114^{\circ} 20^{\prime} \mathrm{O}$ (Fig. 1). Las barras que delimitan las lagunas son la de 0 jo de Liebre y San José respectivamente. Las lagunas están separadas por un extenso campo de dunas de origen eólico, tipo barchán, cubriendo un área de $1.21 \times 10^{8} \mathrm{~m}^{2}$ (Inman et al., 1966).

E1 clima es árido y la precipitación muy baja con un

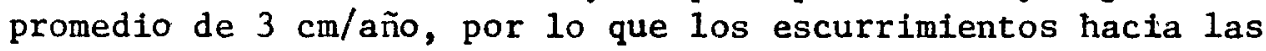
lagunas se pueden considerar despreciables. E1 viento presenta una estacionalidad bien definida. En verano es de mar a tierra y en Invierno de mar a tierra en el dia y de tierra a mar en la noche (Inman et a1., 1966). La sedimentologla de la laguna de Guerrero Negro la presentan Phleger y Ewing (1962) y Phleger (1965). 
La costa de Bahía Vizcaino está constituída por cantiles desde Punta Eugenia hasta donde termina la parte de 1a bahía orientada E-O, mientras que el resto es formado por playas de arena, 1o que permite suponer un alto transporte de sedimentos (arena).

\section{METODOLOGIA}

La información base se obtuvo de la estadística de parámetros de oleaje en mar abierto provenientes del hemisferio norte (estación A) rccopilada por Marine $\Lambda$ dvisers (1961) (Fig. 2).

De este compendio, se seleccionaron cuatro olas significativas por la imposibilidad de operar con todo el espectro del oleaje. Esta selección está fundamentada en que estas presentan el mayor porcentaje de ocurrencia durante el año (Tabla 1).

La batimetría se obtuvo de las cartas de navegación y se elaboraron los diagramas de refracción (para cada una de las olas) siguiendo el método descrito por Wiegel (1964) para obtener la altura de la ola en aguas someras. El criterio de rompiente $s=0.78$ se empleó para determinar la altura de la ola en 1a rompiente (Mc Cowan, 1894; citado por Komar, 1976). Se utilizó la teoría de Airy para obtener la velocidad de fase en aguas someras

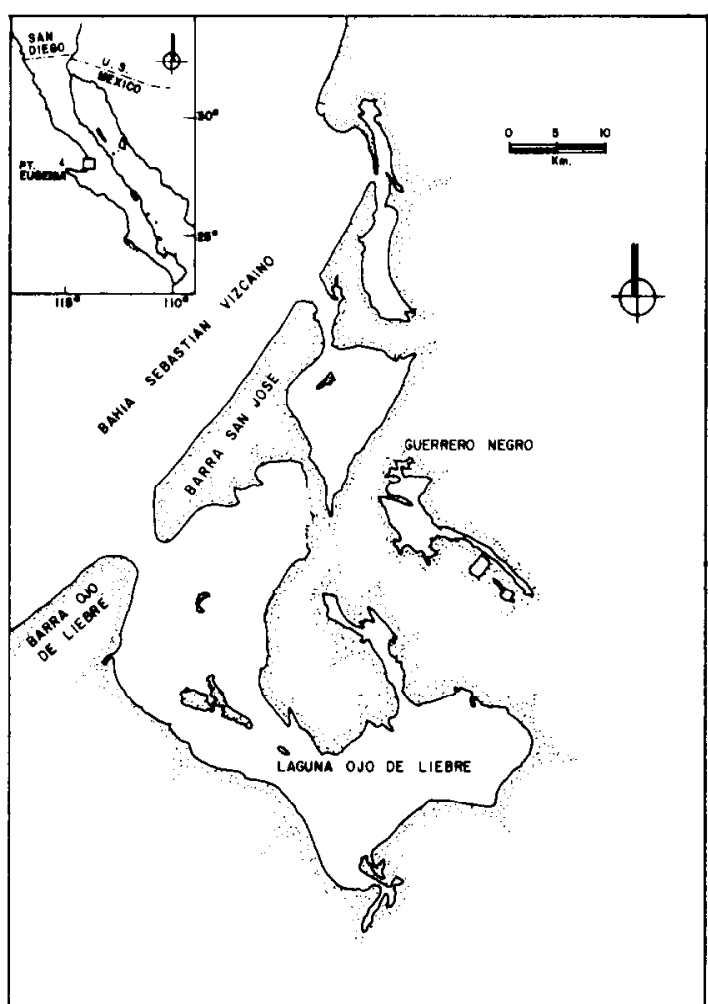

Fig. 1

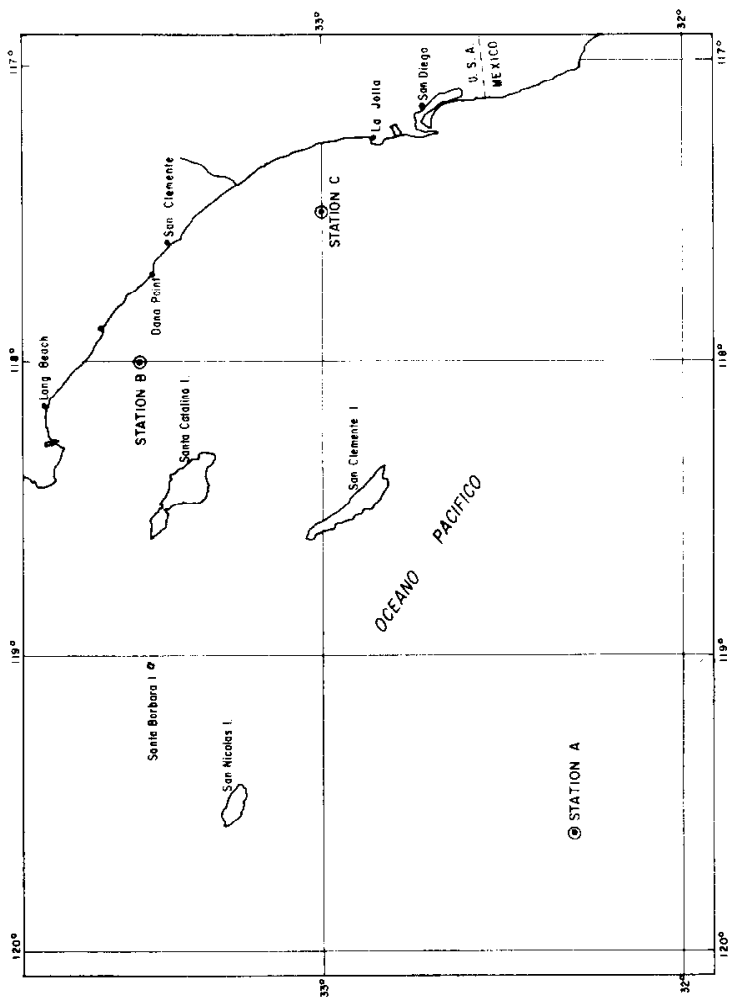

Fig. 2 Localizactón de la estactón A de Marine Advisers (1961). 


$$
\mathrm{C}=\sqrt{\mathrm{gh}},
$$

donde $g$ es la aceleración debida a la gravedad y h la profundidad.

E1 ángulo que forma la cresta de la ola con la linea de playa al momento de romper se determinó de los diagramas de refracción (este parámetro es uno de los más dificiles de obtener en el campo).

E1 transporte potencial de arena en la zona de rompiente se calculó a partir de la ecuación de razón de peso inmerso (dada por Inman y Bagnold (1963))

$$
I=\left(\rho_{s}-\rho\right) \text { ga'sl, }
$$

y del parámetro $P$

$$
P=(E C n)_{r} \operatorname{sen} \alpha_{r} \cos \alpha_{r},
$$

donde $\rho_{S}$ y $\rho$ son la densidad del sedimento y agua respectivamente, $a^{\prime}$ es un factor de correcciôn por espacio vacío, E es la densidad de energía, $n$ es la razón entre la velocidad de grupo y fase, $\alpha$ es el ángulo de rompiente, S1 es el volúmen de sedimento transportado por unidad de tiempo y el subíndice $r$ denota rompiente.

Las ecuaciones (2) y (3) se relacionan mediante

$$
I=k P \text {, }
$$

donde $k$ es un coefeciente adimensional de proporcionalidad. Komar e Inman (1970) determinaron (empîricamente) que esta constante tiene un valor de 0.77 para playas de arena fina de cuarzo. Para el mismo tido de playa. el factor de corrección por espacio vacío (a') es de 0.6 (Komar, 1976). De esta forma, substituyendo (2) y (3) en (4), el volúmen total de arena transportado es

$$
\mathrm{S} 1=6.85 \times 10^{-5}(\mathrm{ECn})_{\mathrm{r}} \operatorname{sen} \alpha_{\mathrm{r}} \cos \alpha_{\mathrm{r}},
$$

donde (ECn) está dada en erg/cm-seg y $\mathrm{s} 1$ en m²/día. En esta realción, ECn fué evaluado a partir de anålisis espectral, i.e., utilizando la raíz cuadrática media de la altura de la ola $\left(\mathrm{H}_{\mathrm{rms}}\right)$. En nuestro caso utilizamos la altura significativa de la ola, por lo tanto, como

$$
2\left(\mathrm{H}_{\mathrm{rms}}\right)^{2}=\left(\mathrm{H}_{\mathrm{s}}\right)^{2} \text {, }
$$

la ecuación (5) se modifica a

$$
S 1=3.425 \times 10^{-}(\mathrm{ECn})_{r} \operatorname{sen} \alpha_{r} \cos \alpha_{r},
$$

para utilizar directamente la altura de la ola signiticativa. 


\section{RESULTADOS Y DISCUSIONES}

Con los datos presentados en la Tabla 1, se construyeron los diagramas de refracciôn de oleaje para la zona de estudio (Figs. 3, 4, 5 y 6). Se observa en general, la tendencia de las ortogonales a diverger hacia la costa, lo cual es de esperarse por la morfología de las bahías (Bascom, 1960). Sin embargo, en el área de la boca de la Laguna Ojo de Liebre se observa una ligera tendencia de las ortogonales a converger debido a las caracterîsticas topográficas del fondo justo frente a la misma. Esta convergencia se presenta para las olas con direcciones de $315^{\circ}$ y $325^{\circ}$ (Figs. 5 y 6).

Las ortogonales en los diagramas de refracciôn se numeraron para facilitar su posterior utilización en la determinación de la altura de rompiente $\left(H_{S}\right)$, profundidad de rompiente (h) y ángulo de incidencia $(\alpha)$ que se presentan en la Tabla 2. El signo negativo se emplea como una convención para indicar, en este caso, que el ángulo de incidencia se abre hacia el so.

Para evaluar el transporte 1itoral en la zona de estudio, se efectuó el promedio de $\mathrm{H}_{\mathrm{S}}$, h y a que se obtuvieron en cada barra (Tabla 3 ).

Como se puede observar en la Tabla 3, el ângulo es siempre positivo (apertura del ángulo hacia el NE) a excepción de la zona cercana a la boca de la Laguna ojo de Liebre. Esto se debe al bajo de arenć en forma de gancho que se 1ocaliza frente a la boca de la laguna, lo que produce que la ola viaje más rápido al NE del mismo, produciendo por 10 tanto, un transporte litoral hacia el so.

Efectuando el cálculo de transporte potencial de arena por cada dirección de aproximación y para cada zona

(Tabla 4), se encuentra que el transporte litoral neto es hacla el $\mathrm{NE}$ con una magnitud del orden de $6 \times 10^{4} \mathrm{~m}^{3} /$ año (Fig. 7).

Por otra parte, la migración de la boca de una laguna costera, rio o estuarto es producida ya sea por la existencia de un transporte litoral neto, o bien por la modificación del curso del rîo que ahî desemboque (Lizárraga-Arciniega, 1972). En el caso de la Laguna Ojo de Liebre, no existe aporte por ríos. De acuerdo a Komar (1976), en la ausencia de otro fenómeno, que no sea el de transporte 1itoral, la boca de una laguna costera tenderá a migrar en dirección de este transporte.

Debido a la dirección del transporte litoral neto obtenido en este trabajo (Fig. 7), más la evidencia de que la boca de la Laguna de Guerrero Negro ha migrado hacia el NE (Phleger, 1965), es posible esperar que la boca de la Laguna ojo de Liebre esté también migrando en la misma dirección. 


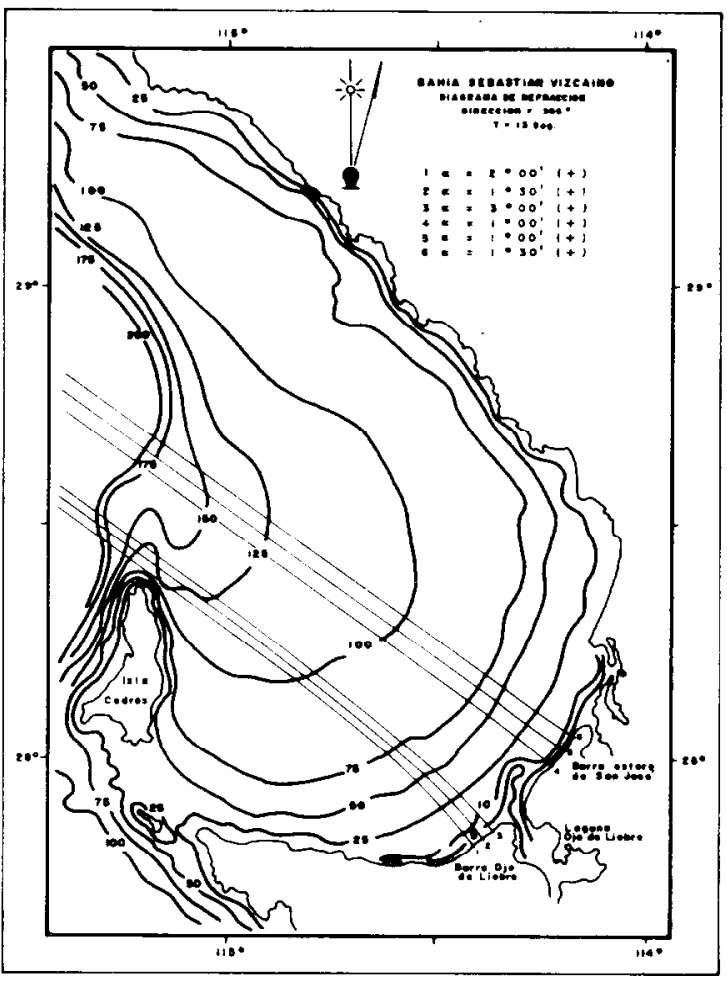

Fig. 3 Diagrama de refracción para el oleaje con dirección de $295^{\circ}$ y periodo de 13 segundos.

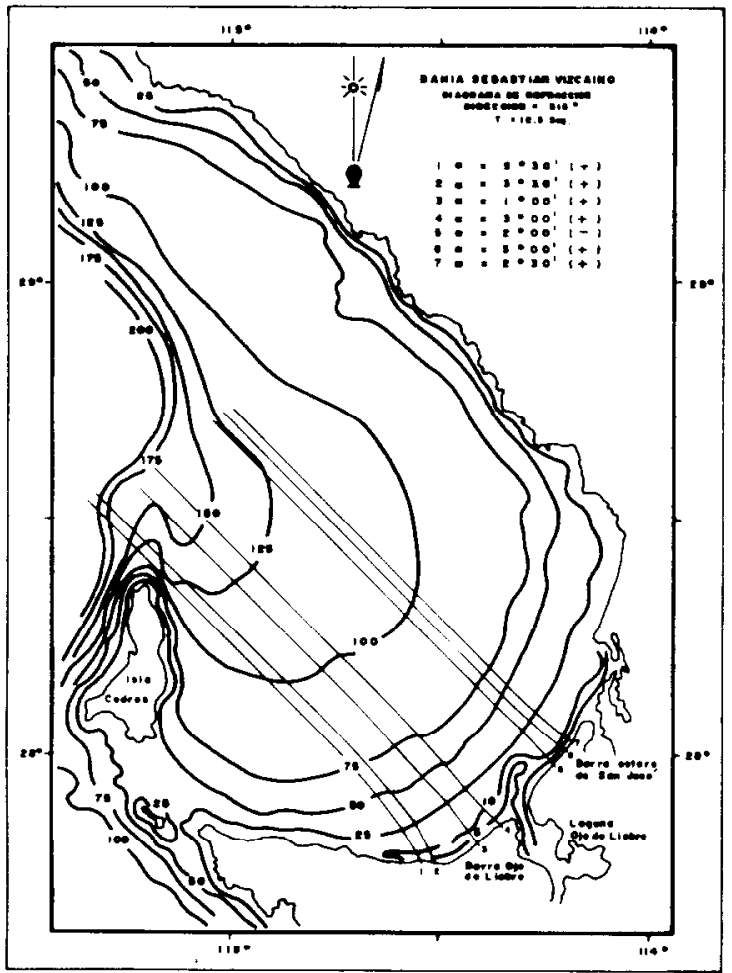

Fig. 5 Diagrama de refracción para el oleaje con dirección de $315^{\circ}$ y perfodo de 12.3 segundos.

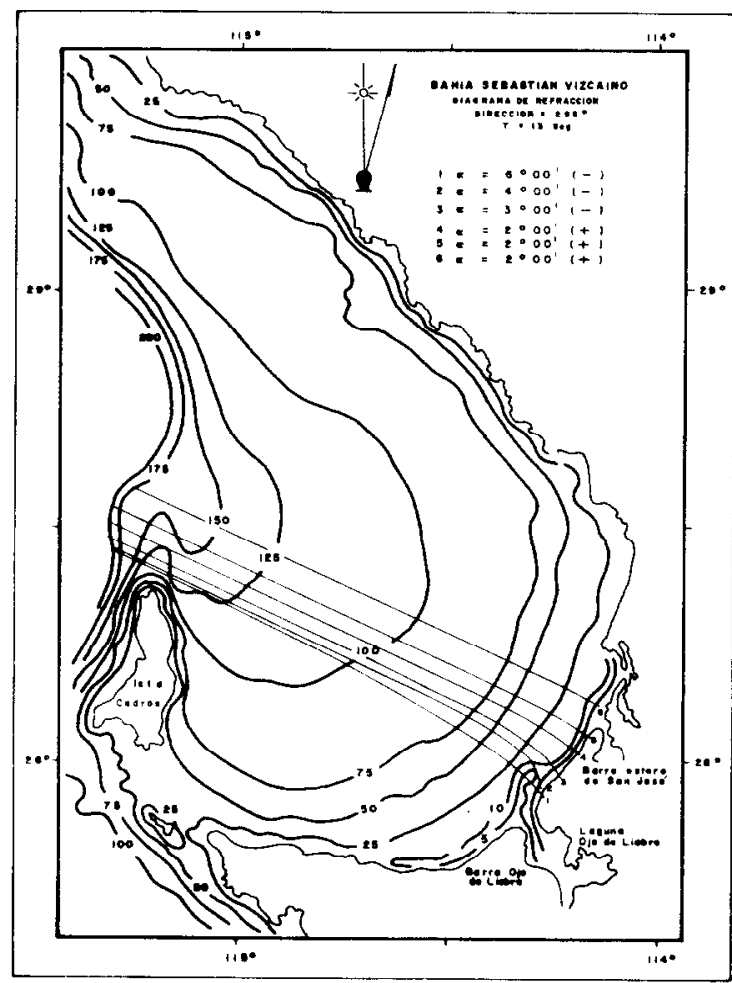

Fig. 4 Dlagrama de refracción para el oleaje con direcciôn de $305^{\circ}$ y perlodo de 13 segundos.

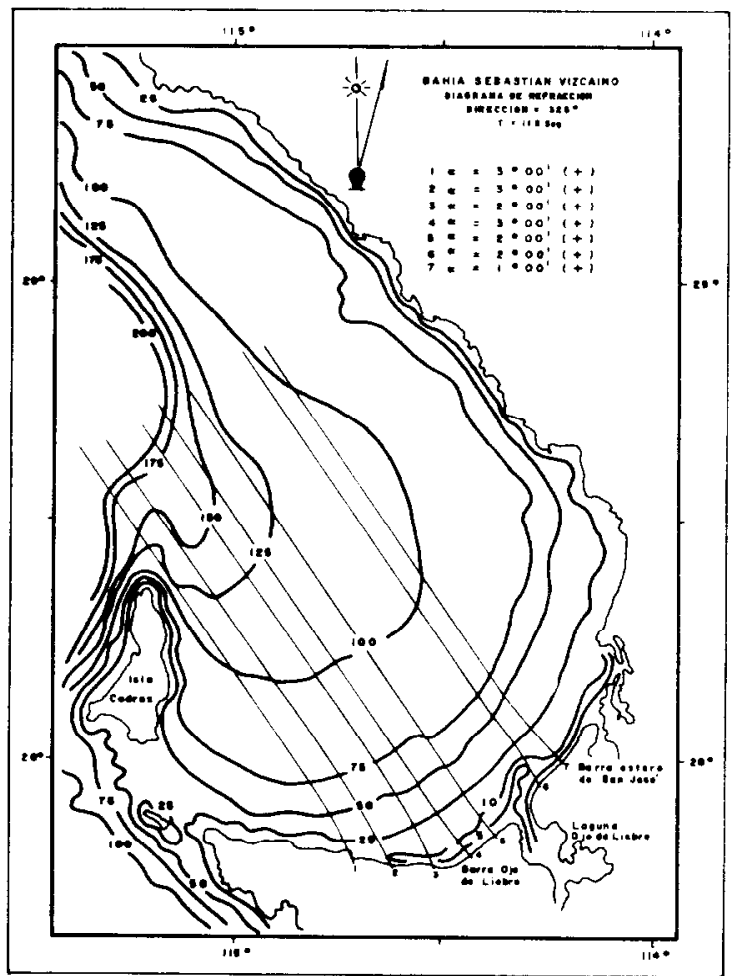

F1g. 6 Diagrama de refracciớn para el oleaje con direcciôn de $325^{\circ}$ y perfodo de 11.5 segundos. 
TABLA 1

$\begin{array}{lllcc}\text { No. de ola } & \mathrm{H}_{\mathrm{O}}(\mathrm{m}) & \mathrm{T}(\mathrm{s}) & \text { dirección } & \text { Frecuencia (\% de1 año) } \\ 1 & 0.81 & 13.0 & 295^{\circ} & 21.4 \\ 2 & 0.80 & 13.0 & 305^{\circ} & 25.2 \\ 3 & 0.80 & 12.3 & 315^{\circ} & 13.7 \\ 4 & 0.94 & 11.5 & 325^{\circ} & 8.3 \\ & & & & \text { Total }=68.6\end{array}$

Tabla 1 Caracteristicas del oleaje típico de tormentas lejanas en aguas profundas (swell del hemisferto norte) en la estación A de Marine Advisers (1961). $H_{0}$ es 1a altura de la ola significativa en aguas profundas, $T$ el período y la dirección es azimutal respecto al norte geogräfico.

TABLA

\begin{tabular}{|c|c|c|c|c|c|}
\hline Dirección & rayos pron. & $\mathrm{H}_{\mathrm{s}}(\mathrm{cm})$ & h $(\mathrm{cm})$ & $\alpha$ & Zona \\
\hline $295^{\circ}$ & $1,2,3$ & 56.0 & 71.5 & -4.30 & A \\
\hline " & $4,5,6$ & 75.0 & 96.0 & 2.00 & B \\
\hline $305^{\circ}$ & $1,2,3$ & 70.0 & 90.0 & 2.17 & C \\
\hline " & $4,5,6$ & 72.5 & 93.0 & 1.17 & B \\
\hline $315^{\circ}$ & $1,2,3,4$ & 71.3 & 91.0 & 3.25 & $\mathrm{C}$ \\
\hline$"$ & 5,6 & 65.0 & 84.0 & -2.00 & A \\
\hline$"$ & 6,7 & 82.0 & 105.0 & 2.75 & B \\
\hline $325^{\circ}$ & $1,2,3,4,5$ & 80.5 & 103.0 & 2.60 & c \\
\hline Ir & 6,7 & 74.0 & 95.0 & 1.50 & B \\
\hline
\end{tabular}

Zona A - Boca ojo de Liebre en barra San José

Zona B - Barra San José

Zona C - Barra Ojo de Liebre

Tabla 3 Promedios de las caracteristicas del oleaje en la zona de rompiente en $1_{\text {as }}$ zonas A, B y C.
TABLA 2

\begin{tabular}{|c|c|c|c|c|}
\hline $\mathrm{Ola}$ & Rayo & $\mathrm{H}_{\mathrm{s}}(\mathrm{cm})$ & $\mathrm{h}(\mathrm{cm})$ & $a\left(^{\circ}\right)$ \\
\hline 1 & $\begin{array}{l}1 \\
2 \\
3 \\
4 \\
5 \\
6\end{array}$ & $\begin{array}{l}62 \\
50 \\
\\
75 \\
74\end{array}$ & $\begin{array}{l}79 \\
64 \\
\\
97 \\
95\end{array}$ & $\begin{array}{r}-6.0 \\
-4.0 \\
-3.0 \\
2.0 \\
2.0 \\
2.0\end{array}$ \\
\hline 2 & $\begin{array}{l}1 \\
2 \\
3 \\
4 \\
5 \\
6\end{array}$ & $\begin{array}{l}73 \\
68 \\
70 \\
75\end{array}$ & $\begin{array}{l}94 \\
87 \\
\\
90 \\
96\end{array}$ & $\begin{array}{l}2.0 \\
1.5 \\
3.0 \\
1.0 \\
1.0 \\
1.5\end{array}$ \\
\hline 3 & $\begin{array}{l}1 \\
2 \\
3 \\
4 \\
5 \\
6 \\
7\end{array}$ & $\begin{array}{l}73 \\
68 \\
73 \\
\\
65 \\
82\end{array}$ & $\begin{array}{r}93 \\
87 \\
93 \\
\\
84 \\
105\end{array}$ & $\begin{array}{r}5.5 \\
3.5 \\
1.0 \\
3.0 \\
-2.0 \\
3.0 \\
2.5\end{array}$ \\
\hline 4 & $\begin{array}{l}1 \\
2 \\
3 \\
4 \\
5 \\
6 \\
7\end{array}$ & $\begin{array}{l}65 \\
89 \\
72 \\
96 \\
74\end{array}$ & $\begin{array}{r}83 \\
114 \\
93 \\
123 \\
\\
95\end{array}$ & $\begin{array}{l}3.0 \\
3.0 \\
2.0 \\
3.0 \\
2.0 \\
2.0 \\
1.0\end{array}$ \\
\hline
\end{tabular}

Tabla 2 Caracteristicas del oleaje en la zona de rorapiente. $H_{s}$ es la altura de la ola significativa en la rompiente, h la profundidad a la que rompe la ola y a el ángulo que forma la cresta con la linea de playa.

TABLA 4

\begin{tabular}{|c|c|c|c|}
\hline Dirección & $\mathrm{S} 1\left(\mathrm{~m}^{3} / \mathrm{d} \mathrm{I} a\right)$ & \# de dias & $S\left(m^{3} / a n ̃ o\right)$ \\
\hline $295^{\circ}$ & -270 & 78.16 & $-21,105$ \\
\hline " & 260 & " & 20,320 \\
\hline $305^{\circ}$ & 240 & 92.04 & 22,090 \\
\hline " & 140 & " & 12,885 \\
\hline $315^{\circ}$ & 370 & 50.04 & 18,515 \\
\hline " & -180 & $"$ & $-9,005$ \\
\hline$n$ & 445 & $"$ & 22,270 \\
\hline $325^{\circ}$ & 405 & 30.32 & 12,280 \\
\hline " & 190 & $"$ & 5,760 \\
\hline
\end{tabular}

Tabla 4 Transporte litoral a lo Iargo de la playa correspondientes a las zonas de la Tabla 3. 


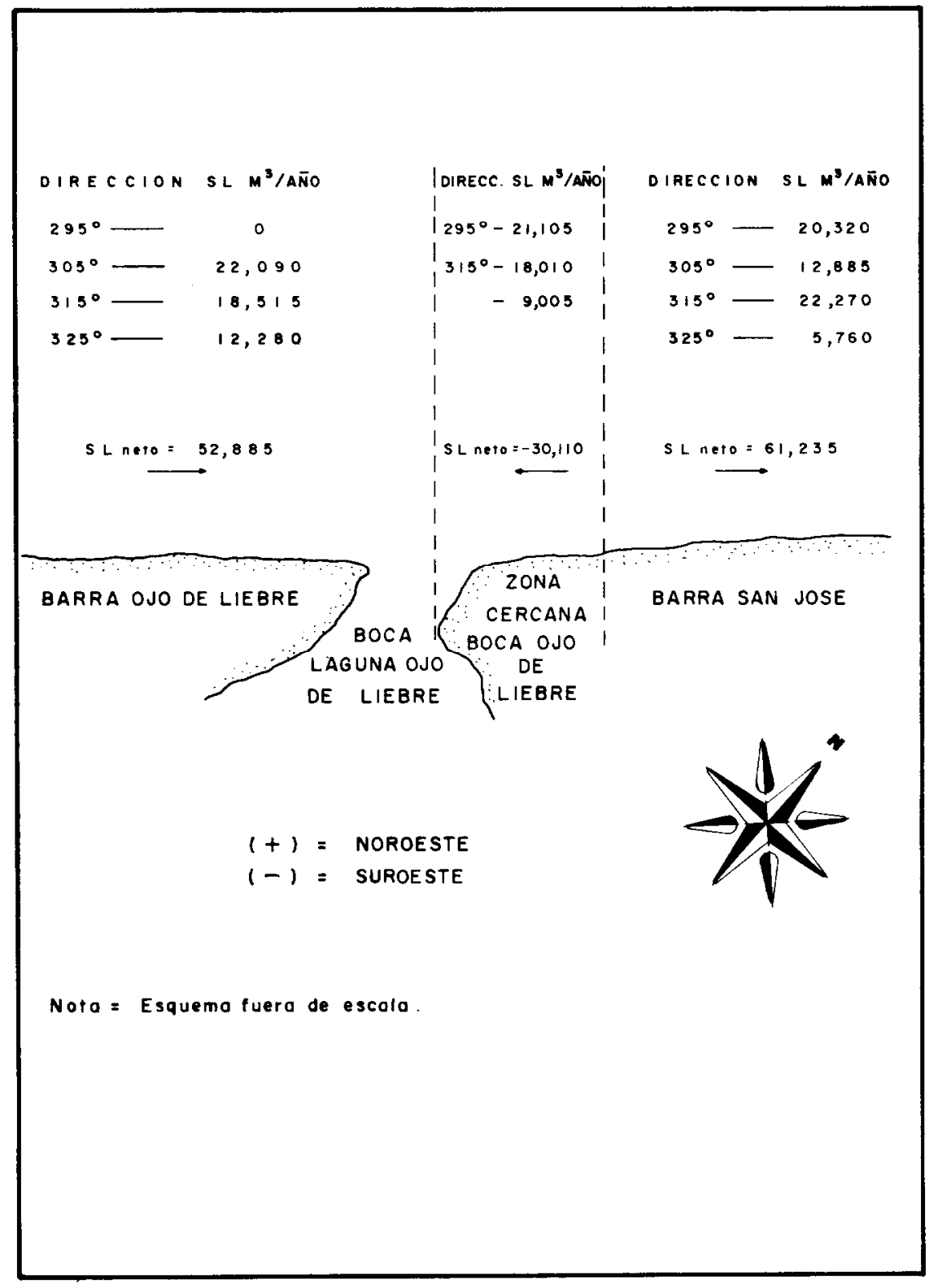

Fig. 7 Esquematización del ấrea de estudio y transporte litoral para cada sera (A, B y C).

Esto se fundamenta en el hecho de que ambas bocas están muy cerca, por 1o que se puede inferir un comportamiento dinámico similar. Además, frente a la boca de la Laguna 0jo de Liebre, se observa claramente un bajo (en forma de gancho) que se extiende hacia el NE (en la misma dirección del transporte neto estimado). Esta evidencia apoya la idea, es decir, se ve una depositación en la parte so de la boca, que con el transporte al NE de la boca sin el aporte del So, producirá una erosión al $\mathrm{NE}$ de la boca. Esto dará lugar a la migración de la misma. 
Estudios previos indican que la barra de San José ha crecido hacia el mar $1.6 \mathrm{~km}$ en 1800\$200 años (Phleger y Ewing, 1962) y que existe un transporte eôlico de $23 \mathrm{~m} / \mathrm{año} / \mathrm{m}$ de playa hacia las lagunas (Inman, et al., 1966). Este transporte eólico implica una descarga de $45 \times 10^{4} \mathrm{~m}^{3} /$ año en toda la barra (i.e., $20 \mathrm{kms}$ de extensión). Como sc ve, aún con la pérdida de arena a las lagunas, las barras siguen ganando terreno al mar, lo que implica la necesidad de un fuerte aporte de material. Este hecho podría ser una indicación de que el volúmen de arena aquí cuantificado sea subestimado, sin embargo, este transporte es a lo largo de la playa y, para esta zona en particular, el que principalmente dará lugar al avance o retroceso de la línea de playa será el perpendicular a la misma. Lo contrario, es decir, el transporte paraleìo a la playa también puede cambiar la línea de playa, sin embargo, Inman et al (1966) muestra que la apatita contenida en la arena de las barras provienen de aguas profundas de la bahía, lo que refuerza el hecho de que el transporte perpendicular a la playa es el principal responsable del avance de la línea de playa.

\section{CONCLUSIONES Y RECOMENDACIONES}

En este trabajo se encontró que frente a las barras de las lagunas de 0 jo de Liebre y Guerrero Negro existe un transporte neto a lo largo de la playa del orden de $6 \times 10^{4} \mathrm{~m}^{3} /$ año. Este transporte litoral neto es en el sentido $\mathrm{NE}$.

E1 sentido del transporte, además de ccnsiderar la migración de la boca de la Laguna de Guerrero Negro, parece implicar un avance de la boca de la Laguna Ojo de Liebre en sentido NE.

La evaluación aquî presentada represenca una primera aproximación del transporte real del área debido a que:

1) Se supone que el oleaje considerado a partir de la estación A de Marine Advisers (1961) 11ega a aguas profundas frente a Bahía Vizcaíno con las mismas caracteristicas.

2) Se estima un transporte a partir del $69 \%$ de ocurrencia del oleaje total del hemisferio norte.

3) Se considera que el oleaje de tormenta lejana del hemisferio sur, produce un transporte litoral despreciable y en la misma dirección, debido a las características morfológicas y batimétricas de la bahỉa.

Un estudio más completo que tome en cuenta el transporte debido al oleaje local, así como la cuantificación del aporte de arena perpendicular a la playa, será de gran utilidad. Asimismo, se manifiesta la necesidad de datos de oleaje (altura, dirección y período) frente a las costas de Baja California. 
AGRADECIMIENTOS

Agradecemos los comentarios y sugerencias de los conpañeros Cuauhtémoc Nava, Adolfo González y Francisco Ocampo, asî como la traducciôn temporal al inglés por George Hemingway del escrito para el valioso referiato anónimo. Sus críticas y comentarios fueron mu importantes para las correcciones finales de este trabajo.

\section{LITERATURA CITADA}

BASCOM, W., 1960. Beaches. Copia del Scientific American No. 845 .

INMAN, D.L. y R.A. Bagnold, 1963. Littoral processes. In the Sea ed. M.N. Hill Interscience, New York, 3:529-533.

INMAN, D.L., G.C. Ewing y J.B. Corliss, 1966. Coasta1 Sand Dunes of Guerrero Negro, Baja California, Mexico. Geo1. Soc. Am. Bul1., 77:787-802.

KOMAR, P.D. y D.L. Inman, 1970. Longshore sand transport on beaches. Jour. Geop. Res., 75(30):591427.

KOMAR, P.D., 1971. The mechanics of sand transport on beaches. Jour. Geop. Res., 76:713-721.

KOMAR, P.D., 1976. Beach processes and sedimentation. PrenticeHa11, Englewood Cliffs, N.J., 532 pp.

LIZARRAGA-ARCINIEGA, J.R., 1972. Estudio de los movimientos cîclicos de la playa en la Bahía Todos Santos, B.C., por medio de secciones transversales. Tésis de Licenciatura, Escuela Superior de Ciencias Marinas, U.A.B.C., Ensenada, B.C.

MARINE Advisers, 1961. A statistical survey of ocean waves characteristics in Southern California waters. U.S. Army Corps of Engineers Contract, No. DA-04-353 CIVENIG 60-37.

BRIEN, P.M. y L. Zeevaert, 1969. Design of a small island inlet. Proceedings of the $11 \mathrm{th}$ Conference of Coastal Engineering. 1242-1257.

PHLEGER, F.B. y G.C. Ewing, 1962. Sedimentology and oceanography of coastal lagoons in Baja California, Mexico. Geol. Soc. Am. Bull., 73:154-182.

PHLEGER, $\overrightarrow{\bar{F}}$. B., 1965. Sedimentology of Guerrero Negro Lagoon, Baja California, Mexico. Contribution No. 48 Marine Foraminifera Laboratory. Contribution from SIO.

WIEGEL, R.L., 1964. Oceanographic Engineering. Prentice-Ha11, Englewood Cliffs, N.J., 429 pp. 